\title{
Morphological characteristics of the plantar calcaneocuboid ligaments
}

\author{
Mutsuaki Edama ${ }^{1,2^{*}} \mathbb{D}$, Tomoya Takabayashi ${ }^{1}$, Hirotake Yokota ${ }^{1}$, Ryo Hrabayashi ${ }^{1}$, Chie Sekine $^{1}$ and Ikuo Kageyama ${ }^{2}$
}

\begin{abstract}
Background: The aim of this study was to clarify the differences in morphological features between the long plantar ligament (LPL) and the short plantar ligament (SPL).

Methods: This investigation examined 50 legs from 25 Japanese cadavers. The LPL and SPL of each leg were classified into one of three types based on the shape and number of fiber bundles. Then, fiber bundle length, fiber bundle width, and fiber bundle thickness were measured.

Results: The LPL was rectangular in shape (Type I) in 12\%, hourglass shape (Type II) in 62\%, and triangular in shape (Type III) in 26\%. The SPL was a single fiber bundle (Type I-a) in 26\%, a surface fiber bundle and a deep fiber bundle (Type I-b) in 60\%, and a surface fiber bundle (medial and lateral) and a deep fiber bundle (Type II) in 14\%.

Regarding the morphological characteristics, there were no significant differences among the types in the LPL, but there were differences between types and between surface and deep fiber bundles in the SPL.

Conclusions: For the LPL, the hourglass shape is the most common type. However, there appeared to be no functional difference due to the difference in the shape of the $L P L$, since there were no significant differences among the types in the LPL. For the SPL, there were types of single, double and triple fiber bundles; there may be functional differences based on the number of fiber bundles and between superficial and deep fibers.
\end{abstract}

Keywords: Long plantar ligament, Short plantar ligament, Calcaneocuboid joint

\section{Background}

Isolated injuries involving the calcaneocuboid joint are rare and frequently overlooked. Indeed, these injuries are a cause of symptoms after an inversion injury of the foot, and the resulting clinical manifestations may mimic a lateral ankle ligament injury, which is more common [1-4]. These injuries predominantly affect young and active persons and are of significant economic importance $[1,2]$. Furthermore, patients with injuries involving the calcaneocuboid joint require different treatment approaches than those with the more frequently occurring lateral ankle ligament injury [1]. With a greater

\footnotetext{
* Correspondence: edama@nuhw.ac.jp

${ }^{1}$ Institute for Human Movement and Medical Sciences, Niigata University of Health and Welfare, Shimami-cho 1398, Kita-ku, Niigata City 950-3198, Japan ${ }^{2}$ Department of Anatomy, School of Life Dentistry at Niigata, Nippon Dental University, Niigata, Japan
}

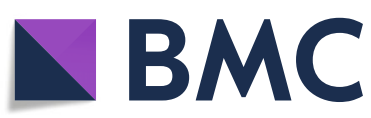

understanding of the morphological characteristics of the ligament, an accurate and timely diagnosis can be made, preventing late sequelae from developing and obviating complicated surgical procedures.

The calcaneocuboid joint is formed by the quadrilateral facets of the calcaneus and cuboid bones and the capsule, which is reinforced by ligaments. The four ligaments connecting the calcaneus and cuboid are: the medial calcaneocuboid ligament, a component of the bifurcate ligament; the dorsolateral calcaneocuboid ligament; the plantar calcaneocuboid ligament, or short plantar ligament; and the long plantar ligament $[5,6]$. These ligaments play a major role in supporting the medial and lateral longitudinal arches [7-9]. However, morphological characteristics and the functional roles of the plantar calcaneocuboid ligaments have not been fully considered.

(c) The Author(s). 2021 Open Access This article is licensed under a Creative Commons Attribution 4.0 International License, which permits use, sharing, adaptation, distribution and reproduction in any medium or format, as long as you give appropriate credit to the original author(s) and the source, provide a link to the Creative Commons licence, and indicate if changes were made. The images or other third party material in this article are included in the article's Creative Commons licence, unless indicated otherwise in a credit line to the material. If material is not included in the article's Creative Commons licence and your intended use is not permitted by statutory regulation or exceeds the permitted use, you will need to obtain permission directly from the copyright holder. To view a copy of this licence, visit http://creativecommons.org/licenses/by/4.0/ The Creative Commons Public Domain Dedication waiver (http://creativecommons.org/publicdomain/zero/1.0/) applies to the data made available in this article, unless otherwise stated in a credit line to the data. 
Previous studies $[5,6,10]$ referred to the plantar calcaneocuboid ligament that was then subdivided into the long plantar ligament (LPL) and the short plantar ligament (SPL); the SPL is also known as the plantar calcaneocuboid ligament. It is generally agreed that the LPL attaches posteriorly to the inferior surface of the calcaneus between the posterior and anterior tubercles [5]. It has been reported that the superficial fibers insert into the bases of the second to fourth metatarsals (MTs) and not the distal cuboid. Previous studies suggested that the superficial fibers insert variably to the metatarsal bone $[6,11]$. One study [6] reported that, in all 59 specimens examined, the LPL had an hourglass shape, and structural variations were observed in the LPL in $20.3 \%$ (12 $\mathrm{ft}$ ), in the form of medial twisting fibers in $11.8 \%$ (7 ft), lateral twisting fibers in $3.3 \%$ ( $2 \mathrm{ft}$ ), and additional bands in $5 \%$ ( $3 \mathrm{ft}$ ). In another study of $10 \mathrm{ft}$, the shape of the LPL was hourglass to rectangular in 100\% [10]. The following morphological data have been reported: length

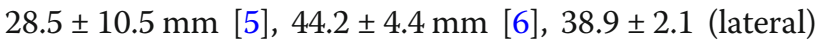
$\mathrm{mm}$ [10], and 61.4 \pm 24.4 (medial) $\mathrm{mm}$ [10]; and width $10.7 \pm 2.8 \mathrm{~mm}$ [5], $10.9 \pm 2.8 \mathrm{~mm}$ [6], and $12.2 \pm 1.1 \mathrm{~mm}$ [10].

The SPL passes anteromedially as a widening band from the anterior tubercle of the calcaneus to attach to the plantar surface of the cuboid posterior to the ridge for the tendon of peroneus longus, and it is often described as blending with and reinforcing the calcaneocuboid joint capsule $[5,6,10]$. Some displayed superficial and deep bands, and in $23 \mathrm{ft}$ (39\%) of the 59 specimens, the deep band had a distinct attachment to the calcaneus [6]. The overall shape and arrangement of the SPL was triangular in $21 \mathrm{ft}(35 \%)$, rectangular in $34 \mathrm{ft}(59 \%)$, and trapezoidal in $4 \mathrm{ft}(6 \%)$. In addition, an extra band or bands were also observed in $19 \mathrm{ft}$ (32\%), in two of which the extra bands were also twisted [6]. In another study, every SPL had at least two bands: superficial and deep [10]. A rectangular or slightly triangular superficial band was seen in $6 \mathrm{ft}(60 \%)$, and in the remaining $4 \mathrm{ft}(40 \%)$, it was triangular with small separate bundles [10]. The following morphological data were reported: length $18.2 \pm$ $4.3 \mathrm{~mm}$ [5], $19.4 \pm 3.6 \mathrm{~mm}$ [6], and $21.0 \pm 1.9 \mathrm{~mm} \mathrm{[10];}$ width $10.3 \pm 4.4 \mathrm{~mm}$ [6], $11.2 \pm 0.8 \mathrm{~mm}$ [10], and $12.2 \pm$ $3.3 \mathrm{~mm}$ [5]; and thickness $4.8 \pm 0.3 \mathrm{~mm}$ [10]. Thus, there are few morphological reports of the plantar calcaneocuboid ligaments, and there is no consensus.

Therefore, the aim of this study was to clarify the differences in morphological features between the LPL and the SPL.

\section{Materials and methods Cadavers}

This investigation examined 50 legs from 25 Japanese cadavers (mean age at death, $78 \pm 12$ years; 28 sides from men, 22 from women; 25 right sides, 25 left sides) that had been switched to alcohol after placement in $10 \%$ formalin. None showed signs of previous major surgery around the foot or ankle or any relevant deformities, and there was no obvious degeneration in all specimens. This study was approved by the Ethics Committee of our institution (18071).

\section{Methods}

The procedures for dissection of the LPL and SPL are described below. All dissections were performed by the same examiner. Isolated specimens of the leg were created by transection $10 \mathrm{~cm}$ above the ankle. The skin, subcutaneous tissue, and crural fascia were removed, and the peroneus longus and brevis and the tibialis posterior were carefully dissected and inspected. The

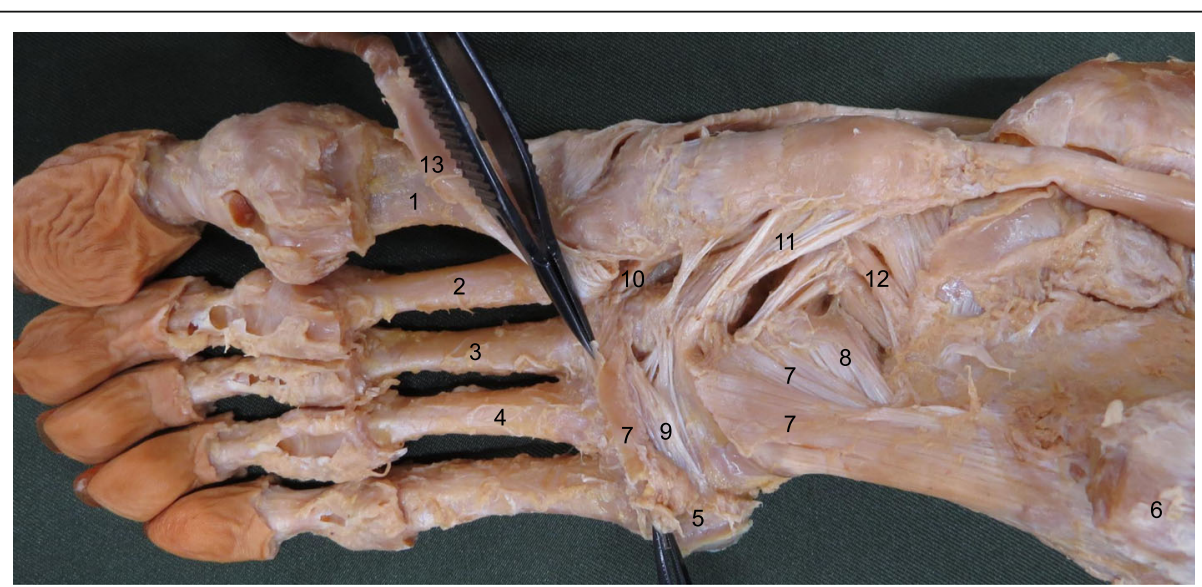

Fig. 1 Dissection procedure for the long plantar ligament: right foot, plantar view 1: First metatarsal, 2: second metatarsal, 3: third metatarsal, 4: fourth metatarsal, 5: base of the fifth metatarsal, 6: calcaneus, 7: long plantar ligament, 8: short plantar ligament, 9: blended fiber bundle of the long plantar ligament, 10: medial cuneiform-metatarsal 2 \& 3 plantar ligament, 11: posterior tibialis, 12: spring ligament (plantar calcaneonavicular ligament), 13: peroneus longus tendon 

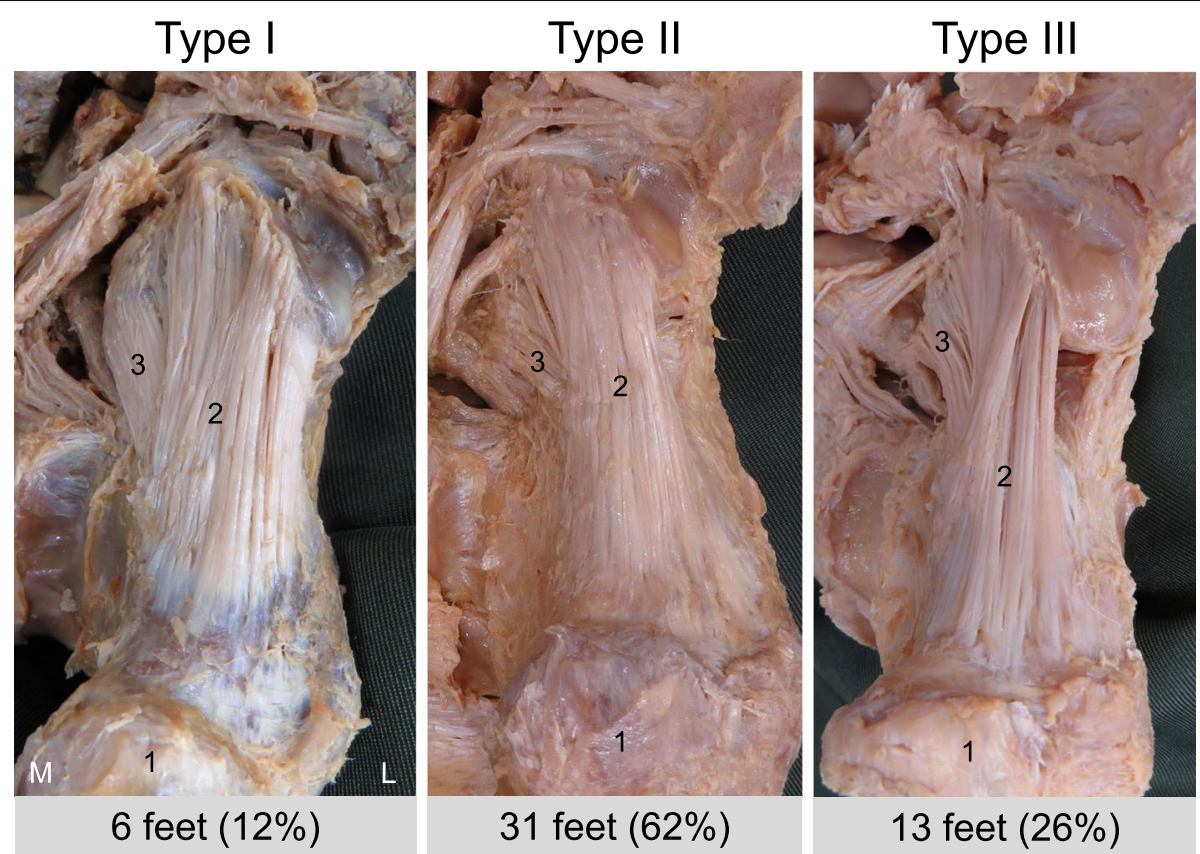

Fig. 2 Classification of the long plantar ligament. Type I: the LPL has a rectangular shape. Type II: the LPL has an hourglass shape. Type III: the LPL has a triangular shape. 1: calcaneus, 2: long plantar ligament, 3: short plantar ligament, L: lateral, M: medial

plantar aponeurosis was meticulously dissected from the flexor digitorum brevis and removed. The first, second, and third plantar layers (flexor digitorum brevis, flexor digitorum longus, flexor hallucis longus, lumbricals, plantar quadratus, abductor hallucis, abductor digiti minimi) were then dissected and excised. Upon reaching the fourth layer, the peroneus longus tendon was once again identified behind the lateral malleolus and then followed into the plantar aspect of the foot. In all specimens, the LPL formed the roof of the peroneus longus canal, blending with the canal before inserting on the metatarsal bases. To clarify the ligaments, the plantar muscles were dissected and excised (Fig. 1). The LPL and SPL of each leg were then classified into one of the three types based on the morphological characteristics and the number of fiber bundles.

Fiber bundle length, fiber bundle width, and fiber bundle thickness were measured for the LPL and the SPL. Fiber bundle length, fiber bundle width, and fiber bundle thickness were measured in the central portions of the LPL and SPL using calipers (Digital Caliper, Shinwa, Niigata, Japan). All measurements were made by the same examiner, with each site measured three times, and the mean value and standard deviation were then calculated.

\section{Statistical analysis}

Statistical analyses were performed using SPSS (version 24.0, SPSS Japan Inc., Tokyo, Japan). The chi-squared test was used for comparisons between men and women and between right and left sides in the classifications based on differences in the type. Comparisons of fiber bundle length, fiber bundle width, and fiber bundle thickness in each type were made with an unpaired $t$ test, one-way repeated measures analysis of variance (ANOVA), and Bonferroni's method. Comparisons of fiber bundle length, fiber bundle width, and fiber bundle thickness among types were made with a paired $t$-test. The level of significance was $5 \%$.

\section{Results}

Classification of the long plantar ligament

Using the classification based on differences in the shape, there were three types: Type I, Type II, and Type III. The types were as follows: Type I, the LPL was a rectangular shape; Type II, the LPL was an hourglass shape; and Type III, the LPL was a triangular shape. Type I was seen in $6 \mathrm{ft}(12 \%)$, Type II in $31 \mathrm{ft}(62 \%)$, and Type III in $13 \mathrm{ft}$ (26\%) (Fig. 2).

There were no significant differences between males and females and between left and right sides (Table 1).

Table 1 Comparison of classifications of the long plantar ligament by sex and laterality

\begin{tabular}{llll}
\hline & Type I & Type II & Type III \\
\hline Male $(n=28)$ & $3(6)$ & $19(38)$ & $6(12)$ \\
Female $(n=22)$ & $3(6)$ & $12(24)$ & $7(14)$ \\
Right $(n=25)$ & $3(6)$ & $16(32)$ & $6(12)$ \\
Left $(n=25)$ & $3(6)$ & $15(30)$ & $7(14)$ \\
\hline
\end{tabular}

Values are reported as number of specimens (\%) 

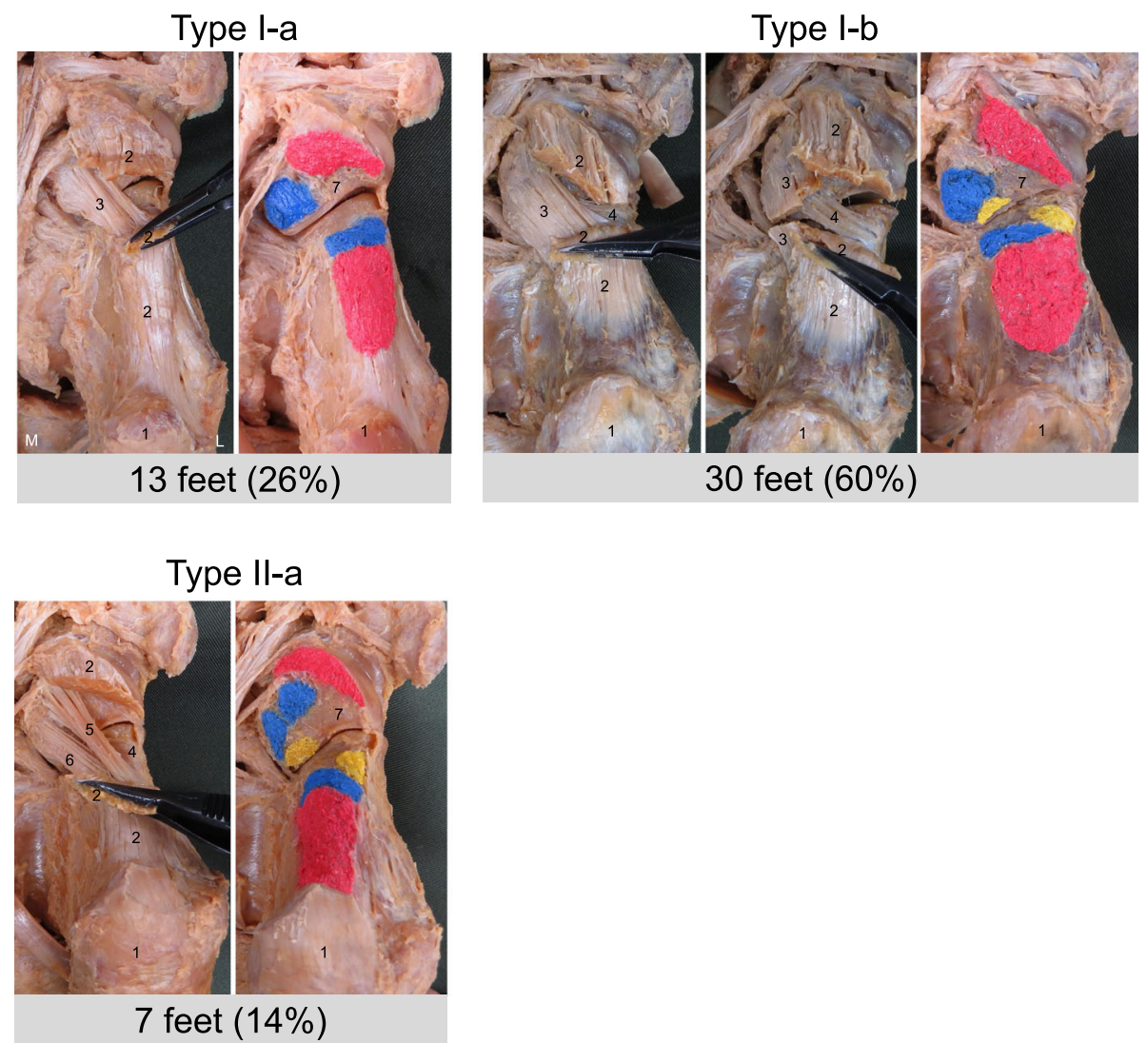

Fig. 3 Classification of the short plantar ligament. Type I-a: the SPL is a superficial (single) fiber bundle. Type I-b: the SPL is a superficial fiber bundle and a deep fiber bundle. Type II: the SPL is a superficial fiber bundle (medial and lateral) and a deep fiber bundle. 1: calcaneus, 2: long plantar ligament, 3: the superficial fiber bundle of the short plantar ligament, 4: the deep fiber bundle of the short plantar ligament, 5: superficial fiber bundle (lateral) of the short plantar ligament, 6: superficial fiber bundle (medial) of the short plantar ligament, 7: cuboid, L: lateral, M: medial, Red area: the footprint of the long plantar ligament at the calcaneus and cuboid. Blue area: the footprint of the superficial fiber bundle of the short plantar ligament at the calcaneus and cuboid. Yellow area: the footprint of the deep fiber bundle of the short plantar ligament at the calcaneus and cuboid

\section{Classification of the short plantar ligament}

Using the classification based on differences in the number of fiber bundles, there were three types: Type I-a, Type I-b, and Type II. The types were as follows: Type I-a, the SPL was a single (superficial) fiber bundle; Type I-b, the SPL was a surface fiber bundle and a deep fiber bundle; Type II, the SPL was a surface fiber bundle (medial and lateral) and a deep fiber bundle. Type I-a was seen in $13 \mathrm{ft}$ (26\%), Type I-b in $30 \mathrm{ft}(60 \%)$, and Type II in $7 \mathrm{ft}(14 \%)$ (Fig. 3).

Table 2 Comparison of classifications of the short plantar ligament by sex and left and right sides

\begin{tabular}{llll}
\hline & Type I-a & Type I-b & Type II \\
\hline Male $(n=28)$ & $8(16)$ & $16(32)$ & $4(8)$ \\
Female $(n=22)$ & $5(10)$ & $14(28)$ & $3(6)$ \\
Right $(n=25)$ & $7(14)$ & $14(28)$ & $4(8)$ \\
Left $(n=25)$ & $6(12)$ & $16(32)$ & $3(6)$ \\
\hline
\end{tabular}

Values are reported as number of specimens (\%)
Every SPL was rectangular in shape. The deep fiber band that reinforced the calcaneocuboid joint capsule was identified with a distinct attachment to the calcaneus and showed a more oblique orientation.

There were no significant differences between males and females and between left and right sides (Table 2).

\section{Morphological characteristics of the long plantar ligament (Table 3)}

There were no significant differences among the types in the LPL.

Table 3 Morphological characteristics of the long plantar ligament

\begin{tabular}{llll}
\hline & Type I & Type II & Type III \\
\hline Length $(\mathrm{mm})$ & $47.4 \pm 3.8$ & $44.3 \pm 5.9$ & $47.9 \pm 5.9$ \\
Width $(\mathrm{mm})$ & $14.7 \pm 1.8$ & $14.2 \pm 2.4$ & $13.7 \pm 2.6$ \\
Depth $(\mathrm{mm})$ & $1.7 \pm 0.3$ & $2.0 \pm 0.5$ & $2.1 \pm 0.6$ \\
\hline
\end{tabular}

Values are means \pm standard deviation 
Table 4 Morphological characteristics of the short plantar ligament

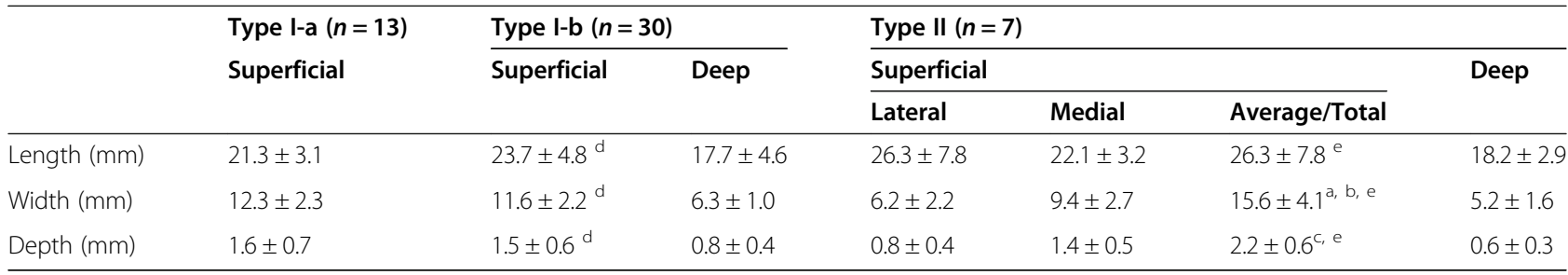

Values are means \pm standard deviation

${ }^{\mathrm{a}} p<0.05$ vs. superficial fiber bundle of Type $\mathrm{I}-\mathrm{a},{ }^{\mathrm{b}} p<0.01$ vs. superficial fiber bundle of Type $\mathrm{I}-\mathrm{b}$

${ }^{c} p<0.05$ vs. deep fiber bundle of Type I-b, ${ }^{d} p<0.01$ vs. deep fiber bundle of Type I-b, ${ }^{e} p<0.01$ vs. deep fiber bundle of Type II

\section{Morphological characteristics of the short plantar ligament (Table 4)}

The superficial fiber bundle was significantly wider in Type II than in Type I-a $(p<0.05)$ and Type I-b $(p<$ $0.01)$. The superficial fiber bundle was significantly thicker in Type II than in Type I-b $(p<0.05)$.

The fiber bundle length, width, and thickness were significantly smaller $(p<0.01)$ in the deep fiber bundles than in the superficial fiber bundles in Type I-b and Type II.

\section{Discussion}

This study clarified the morphological characteristics of the plantar calcaneocuboid ligaments in Japanese cadavers. To the best of our knowledge, there have been no such detailed anatomical studies of these ligaments previously.

For the LPL, the hourglass shape (Type II) was the most common shape, in $62 \%$, followed by the triangular shape (Type III) in 26\% and the rectangular shape (Type I) in $12 \%$. A previous anatomical study [6] reported that the LPL had an hourglass shape in $79.7 \%$, and structural variations (twisting fibers and additional bands) were observed in $20.3 \%$. In another study [10], the shape was hourglass to rectangular in $100 \%$ of the sample. Although the proportions are different, it appears certain that the hourglass shape is the most common type. However, there may be no functional differences due to the differences in the shape of LPL, since there were no significant differences among the types in the LPL.

For the SPL, in the present study, a single fiber bundle (Type I-a) was seen in $26 \%$, and double and triple fiber bundles (Type I-b and Type II) were seen in $74 \%$. In previous studies, the SPL had an extra band or bands in $39 \%$ [6] and 100\% [10]. Although the proportions are different, there are types of single, double and triple fiber bundles. In the present study, every SPL was rectangular in shape. In previous studies, the overall shape of the SPL was triangular in 35\%, rectangular in 59\%, and trapezoidal in 6\% [6], with a rectangular or slightly triangular superficial band in $60 \%$ and a triangular band with small separate bundles in $40 \%$ [10]. The reasons for the difference between the results of the present study and the results of previous studies are unclear and require further investigation. As for the SPL, since morphological characteristics differ between types and between surface and deep fiber bundles, there may be functional differences in the number of fiber bundles and between surficial and deep fibers.

This study has some limitations. First, the morphological characteristics of the LPL and SPL alone were investigated using fixed cadavers, and biomechanical examinations were not conducted. Second, since all of the cadavers were Japanese, it is unclear whether the present findings apply to cadavers of other ethnicities. Thus, future studies are required to evaluate variations according to ethnic origin.

\section{Conclusions}

For the LPL, the hourglass shape was the most common type. In addition, it appears that there may be no functional differences due to the differences in the shape of the LPL, since there were no significant differences among the types in the LPL. For the SPL, there were types of single, double and triple fiber bundles, and every SPL was rectangular in shape. There may be functional differences based on the number of fiber bundles and between superficial and deep fibers. In the future, an in vivo study using ultrasound or MRI examination is needed.

\section{Acknowledgements}

The authors would like to acknowledge and thank those anonymous individuals who generously donated their bodies so that this study could be performed. This study was supported by a Grant-in-Aid for Scientific Research (19 K11358) from the Japanese Society for the Promotion of Science (JSPS) and Commissioned by the Japan Sports Agency (Female Athletes Development and Support Projects 2020).

\section{Authors' contributions}

ME and TT contributed to study design and data collection, and drafted the manuscript; $\mathrm{HY}$ contributed to data analysis and made critical revisions to the manuscript; RH and CS made critical revisions to the manuscript; IK supervised the study, contributed to analysis and interpretation of data, and made critical revisions to the manuscript. All authors read and approved the final manuscript prior to submission.

Funding

None. 


\section{Availability of data and materials}

The data that support the findings of this study are available from the corresponding author upon reasonable request.

\section{Ethics approval and consent to participate}

Informed consent was obtained from the families of all subjects. This study was approved by the ethics committee of the Niigata University of Health and Welfare, Niigata, Japan.

\section{Consent for publication}

Not applicable.

\section{Competing interests}

The authors declare that they have no competing interests.

Received: 2 November 2020 Accepted: 15 December 2020

Published online: 07 January 2021

\section{References}

1. Lohrer H, Arentz S. Calcaneocuboid joint instability: a novel operative technique for anatomic reconstruction. Foot Ankle Int. 2004;25:349-56.

2. Andermahr J, Helling HJ, Maintz D, Mönig S, Koebke J, Rehm KE. The injury of the calcaneocuboid ligaments. Foot Ankle Int. 2000;21:379-84.

3. Leland RH, Marymont JV, Trevino SG, Varner KE, Noble PC. Calcaneocuboid stability: a clinical and anatomic study. Foot Ankle Int. 2001;22:880-4.

4. Jahss $\mathrm{MH}$, Kay BS. An anatomic study of the anterior superior process of the os calcis and its clinical application. Foot Ankle. 1983;3:268-81.

5. Sarrafian SK. Syndesmology. Sarrafian's anatomy of the foot and ankle. 3rd ed. Philadelphia: Lippincott Williams \& Wilkin; 2011. p. 204-6.

6. Ward KA, Soames RW. Morphology of the plantar calcaneocuboid ligaments. Foot Ankle Int. 1997;18:649-53.

7. Dinucci KR, Christensen JC, Dinucci KA. Biomechanical consequences of lateral column lengthening of the calcaneus: part I. long plantar ligament strain. J Foot Ankle Surg. 2004;43:10-5.

8. Otis JC, Deland JT, Kenneally S. Medial arch strain after lateral column lengthening: an in vitro study. Foot Ankle Int. 1999;20:797-802.

9. Huang CK, Kitaoka HB, An KN, Chao EY. Biomechanical evaluation of longitudinal arch stability. Foot Ankle. 1993;14:353-7.

10. Melão L, Canella C, Weber M, Negrão P, Trudell D, Resnick D. Ligaments of the transverse tarsal joint complex: MRI-anatomic correlation in cadavers. AJR Am J Roentgenol. 2009;193:662-71.

11. Hiramoto Y. Variation of the long plantar ligament in Japanese. Okajimas Folia Anat Jpn. 1984;60:401-8.

\section{Publisher's Note}

Springer Nature remains neutral with regard to jurisdictional claims in published maps and institutional affiliations.

Ready to submit your research? Choose BMC and benefit from:

- fast, convenient online submission

- thorough peer review by experienced researchers in your field

- rapid publication on acceptance

- support for research data, including large and complex data types

- gold Open Access which fosters wider collaboration and increased citations

- maximum visibility for your research: over $100 \mathrm{M}$ website views per year

At $\mathrm{BMC}$, research is always in progress.

Learn more biomedcentral.com/submissions 